\title{
A fixed point approach to the Hyers-Ulam stability of a functional equation in various normed spaces
}

\author{
Hassan Azadi Kenary ${ }^{1}$, Sun Young Jang ${ }^{2}$ and Choonkil Park ${ }^{3^{*}}$
}

\footnotetext{
* Correspondence: baak@hanyang. ac.kr

${ }^{3}$ Department of Mathematics, Research Institute for Natural Sciences, Hanyang University, Seoul 133-791, Korea

Full list of author information is available at the end of the article
}

\begin{abstract}
Using direct method, Kenary (Acta Universitatis Apulensis, to appear) proved the Hyers-Ulam stability of the following functional equation

$$
f(m x+n y)=\frac{(m+n) f(x+y)}{2}+\frac{(m-n) f(x-y)}{2}
$$

in non-Archimedean normed spaces and in random normed spaces, where $m, n$ are different integers greater than 1. In this article, using fixed point method, we prove the Hyers-Ulam stability of the above functional equation in various normed spaces. 2010 Mathematics Subject Classification: 39B52; 47H10; 47S40; 46S40; 30G06; 26E30; 46S10; 37H10; 47H40.
\end{abstract}

Keywords: Hyers-Ulam stability, non-Archimedean normed space, random normed space, fuzzy normed space, fixed point method

\section{Introduction}

A classical question in the theory of functional equations is the following: "When is it true that a function which approximately satisfies a functional equation must be close to an exact solution of the equation?" If the problem accepts a solution, then we say that the equation is stable. The first stability problem concerning group homomorphisms was raised by Ulam [1] in 1940. In the following year, Hyers [2] gave a positive answer to the above question for additive groups under the assumption that the groups are Banach spaces. In 1978, Rassias [3] proved a generalization of Hyers' theorem for additive mappings. Furthermore, in 1994, a generalization of the Rassias' theorem was obtained by Găvruta [4] by replacing the bound $\varepsilon\left(\left.\|x\|\right|^{p}+\|y\|^{p}\right)$ by a general control function $\varphi(x, y)$.

The functional equation $f(x+y)+f(x-y)=2 f(x)+2 f(y)$ is called a quadratic functional equation. In particular, every solution of the quadratic functional equation is said to be a quadratic mapping. In 1983, the Hyers-Ulam stability problem for the quadratic functional equation was proved by Skof [5] for mappings $f: X \rightarrow Y$, where $X$ is a normed space and $Y$ is a Banach space. In 1984, Cholewa [6] noticed that the theorem of Skof is still true if the relevant domain $X$ is replaced by an Abelian group and, in 2002, Czerwik [7] proved the Hyers-Ulam stability of the quadratic functional equation.

(c) 2011 Kenary et al; licensee Springer. This is an Open Access article distributed under the terms of the Creative Commons Attribution License (http://creativecommons.org/licenses/by/2.0), which permits unrestricted use, distribution, and reproduction in any medium, provided the original work is properly cited. 
The stability problems of several functional equations have been extensively investigated by a number of authors, and there are many interesting results concerning this problem (see [8-12]).

Using fixed point method, we prove the Hyers-Ulam stability of the following functional equation

$$
f(m x+n y)=\frac{(m+n) f(x+\gamma)}{2}+\frac{(m-n) f(x-y)}{2}
$$

in various spaces, which was introduced and investigated in [13].

\section{Preliminaries}

In this section, we give some definitions and lemmas for the main results in this article.

A valuation is a function $|\cdot|$ from a field $\mathbb{K}$ into $[0, \infty)$ such that, for all $r, s \in \mathbb{K}$, the following conditions hold:

(a) $|r|=0$ if and only if $r=0$;

(b) $|r s|=|r||s|$;

(c) $|r+s| \leq|r|+|s|$.

A field $\mathbb{K}$ is called a valued field if $\mathbb{K}$ carries a valuation. The usual absolute values of $\mathbb{R}$ and $\mathbb{C}$ are examples of valuations.

In 1897, Hensel [14] has introduced a normed space which does not have the Archimedean property.

Let us consider a valuation which satisfies a stronger condition than the triangle inequality. If the triangle inequality is replaced by

$$
|r+s| \leq \max \{|r|,|s|\}
$$

for all $r, s \in \mathbb{K}$ then the function $|\cdot|$ is called a non-Archimedean valuation and the field is called a non-Archimedean field. Clearly, $|1|=|-1|=1$ and $|n| \leq 1$ for all $n \in \mathbb{N}$.

A trivial example of a non-Archimedean valuation is the function $|\cdot|$ taking everything except for 0 into 1 and $|0|=0$.

Definition 2.1. Let $X$ be a vector space over a field $\mathbb{K}$ with a non-Archimedean valuation $|\cdot|$. A function $\|\cdot\|: X \rightarrow[0, \infty)$ is called a non-Archimedean norm if the following conditions hold:

(a) $\|x\|=0$ if and only if $x=0$ for all $x \in X$;

(b) $\|r x||=|r|\| x||$ for all $r \in \mathbb{K}$ and $x \in X$;

(c) the strong triangle inequality holds:

$$
\|x+y\| \leq \max \{\|x\|,\|y\|\}
$$

for all $x, y \in X$. Then $(X,\|\cdot\|)$ is called a non-Archimedean normed space.

Definition 2.2. Let $\left\{x_{n}\right\}$ be a sequence in a non-Archimedean normed space $X$.

(a) The sequence $\left\{x_{n}\right\}$ is called a Cauchy sequence if, for any $\varepsilon>0$, there is a positive integer $N$ such that $\left\|x_{n}-x_{m}\right\| \leq \varepsilon$ for all $n, m \geq N$.

(b) The sequence $\left\{x_{n}\right\}$ is said to be convergent if, for any $\varepsilon>0$, there are a positive integer $N$ and $x \in X$ such that $\left\|x_{n}-x\right\| \leq \varepsilon$ for all $n \geq N$. Then the point $x \in X$ is called the limit of the sequence $\left\{x_{n}\right\}$, which is denote by $\lim _{n \rightarrow \infty} x_{n}=x$. 
(c) If every Cauchy sequence in $X$ converges, then the non-Archimedean normed space $X$ is called a non-Archimedean Banach space.

It is noted that

$$
\left\|x_{n}-x_{m}\right\| \leq \max \left\{\left\|x_{j+1}-x_{j}\right\|: m \leq j \leq n-1\right\}
$$

for all $m, n \geq 1$ with $n>m$.

In the sequel (in random stability section), we adopt the usual terminology, notions, and conventions of the theory of random normed spaces as in [15].

Throughout this article (in random stability section), let $\Gamma^{+}$denote the set of all probability distribution functions $F: \mathbb{R} \cup[-\infty,+\infty] \rightarrow[0,1]$ such that $F$ is left-continuous and nondecreasing on $\mathbb{R}$ and $F(0)=0, F(+\infty)=1$. It is clear that the set $D^{+}=\{F \in$ $\left.\Gamma^{+}: l^{-} F(-\infty)=1\right\}$, where $l^{-} f(x)=\lim _{t \rightarrow x^{-}} f(t)$, is a subset of $\Gamma^{+}$. The set $\Gamma^{+}$is partially ordered by the usual point-wise ordering of functions, i.e., $F \leq G$ if and only if $F(t) \leq G$ $(t)$ for all $t \in \mathbb{R}$. For any $a \geq 0$, the element $H_{a}(t)$ of $D^{+}$is defined by

$$
H_{a}(t)=\left\{\begin{array}{l}
0 \text { if } t \leq a, \\
1 \text { if } t>a .
\end{array}\right.
$$

We can easily show that the maximal element in $\Gamma^{+}$is the distribution function $H_{0}(t)$.

Definition 2.3. [15] A function $T:[0,1]^{2} \rightarrow[0,1]$ is a continuous triangular norm (briefly, a $t$-norm) if $T$ satisfies the following conditions:

(a) $T$ is commutative and associative;

(b) $T$ is continuous;

(c) $T(x, 1)=x$ for all $x \in[0,1]$;

(d) $T(x, y) \leq T(z, w)$ whenever $x \leq z$ and $y \leq w$ for all $x, y, z, w \in[0,1]$.

Three typical examples of continuous $t$-norms are as follows: $T(x, y)=x y, T(x, y)=$ $\max \{a+b-1,0\}$, and $T(x, y)=\min (a, b)$.

Definition 2.4. [16] A random normed space (briefly, $R N$-space) is a triple $(X, \mu, T)$, where $X$ is a vector space, $T$ is a continuous $t$-norm, and $\mu: X \rightarrow D^{+}$is a mapping such that the following conditions hold:

(a) $\mu_{x}(t)=H_{0}(t)$ for all $x \in X$ and $t>0$ if and only if $x=0$;

(b) $\mu_{\alpha x}(t)=\mu_{x}\left(\frac{t}{|\alpha|}\right)$ for all $\alpha \in \mathbb{R}$ with $\alpha \neq 0, x \in X$ and $t \geq 0$;

(c) $\mu_{x+y}(t+s) \geq T\left(\mu_{x}(t), \mu_{y}(s)\right)$ for all $x, y \in X$ and $t, s \geq 0$.

Definition 2.5. Let $(X, \mu, T)$ be an RN-space.

(1) A sequence $\left\{x_{n}\right\}$ in $X$ is said to be convergent to a point $x \in X$ (write $x_{n} \rightarrow x$ as $n \rightarrow \infty)$ if $\lim _{n \rightarrow \infty} \mu_{x_{n}-x}(t)=1$ for all $t>0$.

(2) A sequence $\left\{x_{n}\right\}$ in $X$ is called a Cauchy sequence in $X$ if $\lim _{n \rightarrow \infty} \mu_{x_{n}-x_{m}}(t)=1$ for all $t>0$.

(3) The $R N$-space $(X, \mu, T)$ is said to be complete if every Cauchy sequence in $X$ is convergent.

Theorem 2.1. [15]If $(X, \mu, T)$ is an $R N$-space and $\left\{x_{n}\right\}$ is a sequence such that $x_{n} \rightarrow$ $x$, then $\lim _{n \rightarrow \infty} \mu_{x_{n}}(t)=\mu_{x}(t)$.

Definition 2.6. [17] Let $X$ be a real vector space. A function $N: X \times \mathbb{R} \rightarrow[0,1]$ is called a fuzzy norm on $X$ if for all $x, y \in X$ and all $s, t \in \mathbb{R}$,

(N1) $N(x, t)=0$ for $t \leq 0$;

(N2) $x=0$ if and only if $N(x, t)=1$ for all $t>0$; 
(N3) $N(c x, t)=N\left(x, \frac{t}{|c|}\right)$ if $c \neq 0$;

(N4) $N(x+y, s+t) \geq \min \{N(x, s), N(y, t)\}$

(N5) $N(x,$.$) is a non-decreasing function of \mathbb{R}$ and $\lim _{t \rightarrow \infty} N(x, t)=1$;

(N6) for $x \neq 0, N(x,$.$) is continuous on \mathbb{R}$.

The pair $(X, N)$ is called a fuzzy normed vector space. The properties of fuzzy normed vector space are given in [18].

Example 2.1. Let $(X,\|\cdot\|)$ be a normed linear space and $\alpha, \beta>0$. Then

$$
N(x, t)= \begin{cases}\frac{\alpha t}{\alpha t+\beta\|x\|} t>0, x \in X \\ 0 \quad t \leq 0, x \in X\end{cases}
$$

is a fuzzy norm on $X$.

Definition 2.7. [17] Let $(X, N)$ be a fuzzy normed vector space. A sequence $\left\{x_{n}\right\}$ in $X$ is said to be convergent or converge if there exists an $x \in X$ such that $\lim _{t \rightarrow \infty} N\left(x_{n}-x, t\right)$ $=1$ for all $t>0$. In this case, $x$ is called the limit of the sequence $\left\{x_{n}\right\}$ in $X$ and we denote it by $N-\lim _{t \rightarrow \infty} x_{n}=x$.

Definition 2.8. [17] Let $(X, N)$ be a fuzzy normed vector space. A sequence $\left\{x_{n}\right\}$ in $X$ is called Cauchy if for each $\varepsilon>0$ and each $t>0$ there exists an $n_{0} \in \mathbb{N}$ such that for all $n$ $\geq n_{0}$ and all $p>0$, we have $N\left(x_{n+p}-x_{n}, t\right)>1-\varepsilon$.

It is well known that every convergent sequence in a fuzzy normed vector space is Cauchy. If each Cauchy sequence is convergent, then the fuzzy norm is said to be complete and the fuzzy normed vector space is called a fuzzy Banach space.

Example 2.2. Let $N: \mathbb{R} \times \mathbb{R} \rightarrow[0,1]$ be a fuzzy norm on $\mathbb{R}$ defined by

$$
N(x, t)=\left\{\begin{array}{ll}
\frac{t}{t+|x|} & t>0 \\
0 & t \leq 0
\end{array} .\right.
$$

Then $(\mathbb{R}, N)$ is a fuzzy Banach space.

We say that a mapping $f: X \rightarrow Y$ between fuzzy normed vector spaces $X$ and $Y$ is continuous at a point $x \in X$ if for each sequence $\left\{x_{n}\right\}$ converging to $x_{0} \in X$, then the sequence $\left\{f\left(x_{n}\right)\right\}$ converges to $f\left(x_{0}\right)$. If $f: X \rightarrow Y$ is continuous at each $x \in X$, then $f$ : $X \rightarrow Y$ is said to be continuous on $X$ [19].

Throughout this article, assume that $X$ is a vector space and that $(Y, N)$ is a fuzzy Banach space.

Definition 2.9. Let $X$ be a set. A function $d: X \times X \rightarrow[0, \infty]$ is called a generalized metric on $X$ if $d$ satisfies the following conditions:

(a) $d(x, y)=0$ if and only if $x=y$ for all $x, y \in X$;

(b) $d(x, y)=d(y, x)$ for all $x, y \in X$;

(c) $d(x, z) \leq d(x, y)+d(y, z)$ for all $x, y, z \in X$.

Theorem 2.2. $[20,21]$ Let $(X, d)$ be a complete generalized metric space and $J: X \rightarrow X$ be a strictly contractive mapping with Lipschitz constant $L<1$. Then, for all $x \in X$, either

$$
d\left(J^{n} x, J^{n+1} x\right)=\infty
$$

for all non-negative integers $n$ or there exists a positive integer $n_{0}$ such that

(a) $d\left(J^{n} x, J^{n+1} x\right)<\infty$ for all $n_{0} \geq n_{0}$;

(b) the sequence $\left\{J^{n} x\right\}$ converges to a fixed point $y^{*}$ of $J$;

(c) $y^{*}$ is the unique fixed point of $J$ in the set $Y=\left\{y \in X: d\left(J^{n_{0}} x, y\right)<\infty\right\}$; 
(d) $d\left(y, \gamma^{*}\right) \leq \frac{1}{1-L} d(y$, Jy)for all $y \in Y$.

\section{Non-Archimedean stability of the functional equation (1)}

In this section, using the fixed point alternative approach, we prove the Hyers-Ulam stability of the functional equation (1) in non-Archimedean normed spaces.

Throughout this section, let $X$ be a non-Archimedean normed space and $Y$ a complete non-Archimedean normed space. Assume that $|m| \neq 1$.

Lemma 3.1. Let $X$ and $Y$ be linear normed spaces and $f: X \rightarrow Y$ a mapping satisfying (1). Then $f$ is an additive mapping.

Proof. Letting $y=0$ in (1), we obtain

$$
f(m x)=m f(x)
$$

for all $x \in X$. So one can show that

$$
f\left(m^{n} x\right)=m^{n} f(x)
$$

for all $x \in X$ and all $n \in \mathbb{N}$.

Theorem 3.1. Let $\zeta: X^{2} \rightarrow[0, \infty)$ be a function such that there exists an $L<1$ with

$$
|m| \zeta(x, y) \leq L \zeta(m x, m y)
$$

for all $x, y \in X$. If $f: X \rightarrow Y$ is a mapping satisfying $f(0)=0$ and the inequality

$$
\left\|f(m x+n y)-\frac{(m+n) f(x+y)}{2}-\frac{(m-n) f(x-y)}{2}\right\| \leq \zeta(x, y)
$$

for all $x, y \in X$, then there is a unique additive mapping $A: X \rightarrow Y$ such that

$$
\|f(x)-A(x)\| \leq \frac{L \zeta(x, 0)}{|m|-|m| L} .
$$

Proof. Putting $y=0$ and replacing $x$ by $\frac{x}{m}$ in (2), we have

$$
\left\|m f\left(\frac{x}{m}\right)-f(x)\right\| \leq \zeta\left(\frac{x}{m}, 0\right) \leq \frac{L}{|m|} \zeta(x, 0)
$$

for all $x \in X$. Consider the set

$$
S:=\{g: X \rightarrow Y ; g(0)=0\}
$$

and the generalized metric $d$ in $S$ defined by

$$
d(f, g)=\inf \left\{\mu \in \mathbb{R}^{+}:\|g(x)-h(x)\| \leq \mu \zeta(x, 0), \quad \forall x \in X\right\},
$$

where inf $\varnothing=+\infty$. It is easy to show that $(S, d)$ is complete (see [[22], Lemma 2.1]). Now, we consider a linear mapping $J: S \rightarrow S$ such that

$$
J h(x):=m h\left(\frac{x}{m}\right)
$$

for all $x \in X$. Let $g, h \in S$ be such that $d(g, h)=\varepsilon$. Then we have

$$
\|g(x)-h(x)\| \leq \varepsilon \zeta(x, 0)
$$

for all $x \in X$ and so

$$
\|J g(x)-J h(x)\|=\left\|m g\left(\frac{x}{m}\right)-m h\left(\frac{x}{m}\right)\right\| \leq|m| \varepsilon \zeta\left(\frac{x}{m}, 0\right) \leq|m| \varepsilon \frac{L}{|m|} \zeta(x, 0)
$$


for all $x \in X$. Thus $d(g, h)=\varepsilon$ implies that $d(J g, J h) \leq L \varepsilon$. This means that

$$
d(J g, J h) \leq L d(g, h)
$$

for all $g, h \in S$. It follows from (4) that

$$
d(f, J f) \leq \frac{L}{|m|}
$$

By Theorem 2.2, there exists a mapping $A: X \rightarrow Y$ satisfying the following:

(1) $A$ is a fixed point of $J$, that is,

$$
A\left(\frac{x}{m}\right)=\frac{1}{m} A(x)
$$

for all $x \in X$. The mapping $A$ is a unique fixed point of $J$ in the set

$$
\Omega=\{h \in S: d(g, h)<\infty\} .
$$

This implies that $A$ is a unique mapping satisfying (5) such that there exists $\mu \in(0, \infty)$ satisfying

$$
\|f(x)-A(x)\| \leq \mu \zeta(x, 0)
$$

for all $x \in X$.

(2) $d\left(J^{n} f, A\right) \rightarrow 0$ as $n \rightarrow \infty$. This implies the equality

$$
\lim _{n \rightarrow \infty} m^{n} f\left(\frac{x}{m^{n}}\right)=A(x)
$$

for all $x \in X$.

(3) $d(f, A) \leq \frac{d(f, J f)}{1-L}$ with $f \in \Omega$, which implies the inequality

$$
d(f, A) \leq \frac{L}{|m|-|m| L}
$$

This implies that the inequality (3) holds. By (2), we have

$$
\begin{gathered}
\left\|m^{n} f\left(\frac{m x+n y}{m^{n}}\right)-\frac{m^{n}(m+n) f\left(\frac{x+\gamma}{m^{n}}\right)}{2}-\frac{m^{n}(m-n) f\left(\frac{x-\gamma}{m^{n}}\right)}{2}\right\| \\
\leq|m|^{n} \zeta\left(\frac{x}{m^{n}}, \frac{\gamma}{m^{n}}\right) \leq|m|^{n} \cdot \frac{L^{n}}{|m|^{n}} \zeta(x, \gamma)
\end{gathered}
$$

for all $x, y \in X$ and $n \geq 1$ and so

$$
\left\|A(m x+n y)-\frac{(m+n) A(x+y)}{2}-\frac{(m-n) A(x-y)}{2}\right\|=0
$$

for all $x, y \in X$.

On the other hand

$$
m A\left(\frac{x}{m}\right)-A(x)=\lim _{n \rightarrow \infty} m^{n+1} f\left(\frac{x}{m^{n+1}}\right)-\lim _{n \rightarrow \infty} m^{n} f\left(\frac{x}{m^{n}}\right)=0 .
$$

Therefore, the mapping $A: X \rightarrow Y$ is additive. This completes the proof. $\square$

Corollary 3.1. Let $\theta \geq 0$ and $p$ be a real number with $0<p<1$. Let $f: X \rightarrow Y$ be $a$ mapping satisfying $f(0)=0$ and the inequality 


$$
\left\|f(m x+n y)-\frac{(m+n) f(x+y)}{2}-\frac{(m-n) f(x-y)}{2}\right\| \leq \theta\left(\|x\|^{p}+\|y\|^{p}\right)
$$

for all $x, y \in X$. Then, for all $x \in X$,

$$
A(x)=\lim _{n \rightarrow \infty} m^{n} f\left(\frac{x}{m^{n}}\right)
$$

exists and $A: X \rightarrow Y$ is a unique additive mapping such that

$$
\|f(x)-A(x)\| \leq \frac{|m| \theta|| x||^{p}}{|m|^{p+1}-|m|^{2}}
$$

for all $x \in X$.

Proof. The proof follows from Theorem 3.1 if we take

$$
\zeta(x, y)=\theta\left(\|x\|^{p}+\|y\|^{p}\right)
$$

for all $x, y \in X$. In fact, if we choose $L=|m|^{1-p}$, then we get the desired result. $\square$

Theorem 3.2. Let $\zeta: X^{2} \rightarrow[0, \infty)$ be a function such that there exists an $L<1$ with

$$
\frac{\zeta(m x, m y)}{|m|} \leq L \zeta(x, y)
$$

for all $x, y \in X$. Let $f: X \rightarrow Y$ be a mapping satisfying $f(0)=0$ and (2). Then there is a unique additive mapping $A: X \rightarrow Y$ such that

$$
\|f(x)-A(x)\| \leq \frac{\zeta(x, 0)}{|m|-|m| L} .
$$

Proof. The proof is similar to the proof of Theorem 3.1. $\square$

Corollary 3.2. Let $\theta \geq 0$ and $p$ be a real number with $p>1$. Let $f: X \rightarrow Y$ be a mapping satisfying $f(0)=0$ and (6). Then, for all $x \in X$

$$
A(x)=\lim _{n \rightarrow \infty} \frac{f\left(m^{n} x\right)}{m^{n}}
$$

exists and $A: X \rightarrow Y$ is a unique additive mapping such that

$$
\|f(x)-A(x)\| \leq \frac{\theta \|\left. x\right|^{p}}{|m|-|m|^{p}}
$$

for all $x \in X$.

Proof. The proof follows from Theorem 3.2 if we take

$$
\zeta(x, y)=\theta\left(\|x\|^{p}+\|y\|^{p}\right)
$$

for all $x, y \in X$. In fact, if we choose $L=|2 m|^{p-1}$, then we get the desired result. $\square$

Example 3.1. Let $Y$ be a complete non-Archimedean normed space. Let $f: Y \rightarrow Y$ be a mapping defined by

$$
f(z)=\left\{\begin{array}{l}
z, z \in\{m x+n y:\|m x+n y\|<1\} \cap\{x-y:\|x-y\|<1\} \\
0, \text { otherwise }
\end{array} .\right.
$$

Then one can easily show that $f: Y \rightarrow Y$ satisfies (3.5) for the case $p=1$ and that there does not exist an additive mapping satisfying (3.6). 


\section{Random stability of the functional equation (1)}

In this section, using the fixed point alternative approach, we prove the Hyers-Ulam stability of the functional equation (1) in random normed spaces.

Theorem 4.1. Let $X$ be a linear space, $(Y, \mu, T)$ a complete $R N$-space and $\Phi$ a mapping from $X^{2}$ to $D^{+}\left(\Phi(x, y)\right.$ is denoted by $\left.\Phi_{x, y}\right)$ such that there exists $0<\alpha<\frac{1}{m}$ such that

$$
\Phi_{m x, m y}\left(\frac{t}{\alpha}\right) \leq \Phi_{x, y}(t)
$$

for all $x, y \in X$ and $t>0$. Let $f: X \rightarrow Y$ be a mapping satisfying $f(0)=0$ and

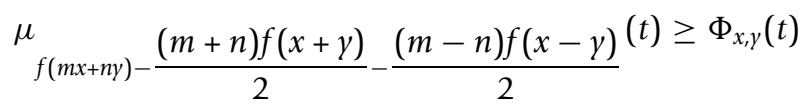

for all $x, y \in X$ and $t>0$. Then, for all $x \in X$

$$
A(x):=\lim _{n \rightarrow \infty} m^{n} f\left(\frac{x}{m^{n}}\right)
$$

exists and $A: X \rightarrow Y$ is a unique additive mapping such that

$$
\mu_{f(x)-A(x)}(t) \geq \Phi_{x, 0}\left(\frac{(1-m \alpha) t}{\alpha}\right)
$$

for all $x \in X$ and $t>0$.

Proof. Putting $y=0$ in (9) and replacing $x$ by $\frac{x}{m}$, we have

$$
\mu_{m f\left(\frac{x}{m}\right)-f(x)}(t) \geq \Phi_{\frac{x}{m}, 0}(t)
$$

for all $x \in X$ and $t>0$. Consider the set

$$
S^{*}:=\{g: X \rightarrow Y ; g(0)=0\}
$$

and the generalized metric $d^{*}$ in $S^{*}$ defined by

$$
d^{*}(f, g)=\inf _{u \in(0,+\infty)}\left\{\mu_{g(x)-h(x)}(u t) \geq \Phi_{x, 0}(t), \quad \forall x \in X, t>0\right\},
$$

where inf $\varnothing=+\infty$. It is easy to show that $\left(S^{*}, d^{*}\right)$ is complete (see [[22], Lemma 2.1]).

Now, we consider a linear mapping $J: S^{*} \rightarrow S^{*}$ such that

$$
\operatorname{Jh}(x):=m h\left(\frac{x}{m}\right)
$$

for all $x \in X$.

First, we prove that $J$ is a strictly contractive mapping with the Lipschitz constant $m \alpha$. In fact, let $g, h \in S^{*}$ be such that $d^{*}(g, h)<\varepsilon$. Then we have

$$
\mu_{g(x)-h(x)}(\varepsilon t) \geq \Phi_{x, 0}(t)
$$

for all $x \in X$ and $t>0$ and so

$$
\begin{aligned}
& \mu_{J g(x)-J h(x)}(m \alpha \varepsilon t)=\mu_{m g\left(\frac{x}{m}\right)-m h\left(\frac{x}{m}\right)}(m \alpha \varepsilon t)=\mu_{g\left(\frac{x}{m}\right)-h\left(\frac{x}{m}\right)}(\alpha \varepsilon t) \\
& \geq \Phi_{\frac{x}{m}, 0}(\alpha t) \\
& \geq \Phi_{x, 0}(t)
\end{aligned}
$$


for all $x \in X$ and $t>0$. Thus $d^{*}(g, h)<\varepsilon$ implies that $d^{*}(J g, J h)<m \alpha \varepsilon$. This means that

$$
d^{*}(J g, J h) \leq \operatorname{mad}(g, h)
$$

for all $g, h \in S^{*}$. It follows from (11) that

$$
d^{*}(f, J f) \leq \alpha .
$$

By Theorem 2.2, there exists a mapping $A: X \rightarrow Y$ satisfying the following:

(1) $A$ is a fixed point of $J$, that is,

$$
A\left(\frac{x}{m}\right)=\frac{1}{m} A(x)
$$

for all $x \in X$. The mapping $A$ is a unique fixed point of $J$ in the set

$$
\Omega=\left\{h \in S^{*}: d^{*}(g, h)<\infty\right\} .
$$

This implies that $A$ is a unique mapping satisfying (12) such that there exists $u \in(0$, $\infty)$ satisfying

$$
\mu_{f(x)-A(x)}(u t) \geq \Phi_{x, 0}(t)
$$

for all $x \in X$ and $t>0$.

(2) $d^{*}\left(J^{n} f, A\right) \rightarrow 0$ as $n \rightarrow \infty$. This implies the equality

$$
\lim _{n \rightarrow \infty} m^{n} f\left(\frac{x}{m^{n}}\right)=A(x)
$$

for all $x \in X$.

(3) $d^{*}(f, A) \leq \frac{d^{*}(f, J f)}{1-m \alpha}$ with $f \in \Omega$, which implies the inequality

$$
d^{*}(f, A) \leq \frac{\alpha}{1-m \alpha}
$$

and so

$$
\mu_{f(x)-A(x)}\left(\frac{\alpha t}{1-m \alpha}\right) \geq \Phi_{x, 0}(t)
$$

for all $x \in X$ and $t>0$. This implies that the inequality (10) holds.

On the other hand

$$
\mu \quad m^{n} f\left(\frac{m x+n y}{m^{n}}\right)-\frac{m^{n}(m+n) f\left(\frac{x+y}{m^{n}}\right)}{2}-\frac{m^{n}(m-n) f\left(\frac{x-y}{m^{n}}\right)}{2}(t) \geq \Phi \frac{x}{m^{n}}, \frac{\gamma}{m^{n}}\left(\frac{t}{m^{n}}\right)
$$

for all $x, y \in X, t>0$ and $n \geq 1$ and so, from (8), it follows that

$$
\Phi_{\frac{x}{m^{n}}, \frac{y}{m^{n}}}\left(\frac{t}{m^{n}}\right) \geq \Phi_{x, y}\left(\frac{t}{m^{n} \alpha^{n}}\right) .
$$

Since

$$
\lim _{n \rightarrow \infty} \Phi_{x, y}\left(\frac{t}{m^{n} \alpha^{n}}\right)=1
$$


for all $x, y \in X$ and $t>0$, we have

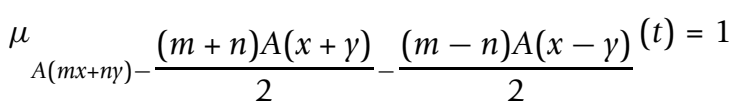

for all $x, y \in X$ and $t>0$.

On the other hand

$$
\begin{aligned}
A(m x)-m A(x) & =\lim _{n \rightarrow \infty} m^{n} f\left(\frac{x}{m^{n-1}}\right)-m \lim _{n \rightarrow \infty} m^{n} f\left(\frac{x}{m^{n}}\right) \\
& =m\left[\lim _{n \rightarrow \infty} m^{n-1} f\left(\frac{x}{m^{n-1}}\right)-\lim _{n \rightarrow \infty} m^{n} f\left(\frac{x}{m^{n}}\right)\right] \\
& =0 .
\end{aligned}
$$

Thus the mapping $A: X \rightarrow Y$ is additive. This completes the proof. $\square$

Corollary 4.1. Let $X$ be a real normed space, $\theta \geq 0$ and let $p$ be a real number with $p$ $>1$. Let $f: X \rightarrow Y$ be a mapping satisfying $f(0)=0$ and

$$
\mu_{f(m x+n y)-\frac{(m+n) f(x+y)}{2}-\frac{(m-n) f(x-y)}{2}}(t) \geq \frac{t}{t+\theta\left(\|x\|^{p}+\|y\|^{p}\right)}
$$

for all $x, y \in X$ and $t>0$. Then, for all $x \in X$,

$$
A(x)=\lim _{n \rightarrow \infty} m^{n} f\left(\frac{x}{m^{n}}\right)
$$

exists and $A: X \rightarrow Y$ is a unique additive mapping such that

$$
\mu_{f(x)-A(x)}(t) \geq \frac{m^{p}\left(1-m^{1-p}\right) t}{m^{p}\left(1-m^{1-p}\right) t+\theta\|x\|^{p}}
$$

for all $x \in X$ and $t>0$.

Proof. The proof follows from Theorem 4.1 if we take

$$
\Phi_{x, y}(t)=\frac{t}{t+\theta\left(\left\|\left.x\right|^{p}+\right\| y \|^{p}\right)}
$$

for all $x, y \in X$ and $t>0$. In fact, if we choose $\alpha=m^{-p}$, then we get the desired result. $\square$

Theorem 4.2. Let $X$ be a linear space, $(Y, \mu, T)$ a complete $R N$-space and $\Phi$ a mapping from $X^{2}$ to $D^{+}\left(\Phi(x, y)\right.$ is denoted by $\left.\Phi_{x, y}\right)$ such that for some $0<\alpha<m$

$$
\Phi_{\frac{x}{m}, \frac{y}{m}}(t) \leq \Phi_{x, y}(\alpha t)
$$

for all $x, y \in X$ and $t>0$. Let $f: X \rightarrow Y$ be a mapping satisfying $f(0)=0$ and

$$
\mu_{f(m x+n y)-} \frac{(m+n) f(x+y)}{2}-\frac{(m-n) f(x-y)}{2}(t) \geq \Phi_{x, y}(t)
$$

for all $x, y \in X$ and $t>0$. Then, for all $x \in X$,

$$
A(x):=\lim _{n \rightarrow \infty} \frac{f\left(m^{n} x\right)}{m^{n}}
$$

exists and $A: X \rightarrow Y$ is a unique additive mapping such that

$$
\mu_{f(x)-A(x)}(t) \geq \Phi_{x, 0}((m-\alpha) t)
$$


for all $x \in X$ and $t>0$.

Proof. The proof is similar to the proof of Theorem 4.1.

Corollary 4.2. Let $X$ be a real normed space, $\theta \geq 0$ and let $p$ be a real number with 0 $<p<1$. Let $f: X \rightarrow Y$ be a mapping satisfying $f(0)=0$ and

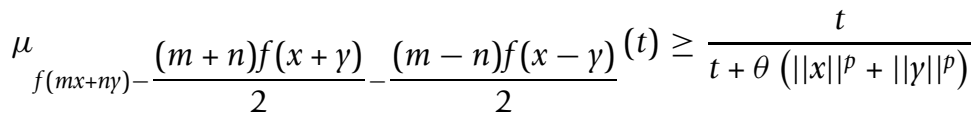

for all $x, y \in X$ and $t>0$. Then, for all $x \in X$,

$$
A(x)=\lim _{n \rightarrow \infty} \frac{f\left(m^{n} x\right)}{m^{n}}
$$

exists and $A: X \rightarrow Y$ is a unique additive mapping such that

$$
\mu_{f(x)-A(x)}(t) \geq \frac{\left(m-m^{p}\right) t}{\left(m-m^{p}\right) t+\theta\|x\|^{p}}
$$

for all $x \in X$ and $t>0$.

Proof. The proof follows from Theorem 4.2 if we take

$$
\Phi_{x, y}(t)=\frac{t}{t+\theta\left(\left\|x||^{p}+\right\| y \|^{p}\right)}
$$

for all $x, y \in X$ and $t>0$. In fact, if we choose $\alpha=m^{p}$, then we get the desired result. $\square$

Example 4.1. Let $(Y, \mu, T)$ be a normed complete RN-space. Let $f: Y \rightarrow Y$ be a mapping defined by

$$
f(z)=\left\{\begin{array}{l}
z, z \in\{m x+n y:\|m x+n y\|<1\} \cap\{x-y:\|x-y\|<1\} \\
0, \text { otherwise }
\end{array} .\right.
$$

Then one can easily show that $f: Y \rightarrow Y$ satisfies (4.6) for the case $p=1$ and that there does not exist an additive mapping satisfying (4.7).

\section{Fuzzy stability of the functional equation (1)}

Throughout this section, using the fixed point alternative approach, we prove the Hyers-Ulam stability of the functional equation (1) in fuzzy normed spaces.

In the rest of the article, assume that $X$ is a vector space and that $(Y, N)$ is a fuzzy Banach space.

Theorem 5.1. Let $\phi: X^{2} \rightarrow[0, \infty)$ be a function such that there exists an $L<1$ with

$$
\varphi\left(\frac{x}{m}, \frac{y}{m}\right) \leq \frac{L}{m} \varphi(x, y)
$$

for all $x, y \in X$. Let $f: X \rightarrow Y$ be a mapping satisfying $f(0)=0$ and

$$
N\left(f(m x+n y)-\frac{(m+n) f(x+y)}{2}-\frac{(m-n) f(x-y)}{2}, t\right) \geq \frac{t}{t+\varphi(x, y)}
$$

for all $x, y \in X$ and all $t>0$. Then the limit

$$
A(x):=N-\lim _{n \rightarrow \infty} m^{n} f\left(\frac{x}{m^{n}}\right)
$$


exists for each $x \in X$ and defines a unique additive mapping $A: X \rightarrow Y$ such that

$$
N(f(x)-A(x), t) \geq \frac{(m-m L) t}{(m-m L) t+L \varphi(x, 0)} .
$$

for all $x, y \in X$ and all $t>0$.

Proof. Putting $y=0$ in (16) and replacing $x$ by $\frac{x}{m}$, we have

$$
N\left(m f\left(\frac{x}{m}\right)-f(x), t\right) \geq \frac{t}{t+\varphi\left(\frac{x}{2}, 0\right)}
$$

for all $x \in X$ and $t>0$. Consider the set

$$
s^{* *}:=\{g: X \rightarrow Y, g(0)=0\}
$$

and the generalized metric $d^{* * *}$ in $S^{* * *}$ defined by

$$
d^{* *}(f, g)=\inf \left\{\mu \in \mathbb{R}^{+}: N(g(x)-h(x), \mu t) \geq \frac{t}{t+\varphi(x, 0)}, \quad \forall x \in X, t>0\right\},
$$

where inf $\varnothing=+\infty$. It is easy to show that $\left(S^{* * *}, d^{* * *}\right)$ is complete (see [[22], Lemma 2.1]).

Now, we consider a linear mapping $J: S^{* * *} \rightarrow S^{* * *}$ such that

$$
J g(x):=m g\left(\frac{x}{m}\right)
$$

for all $x \in X$.

The rest of the proof is similar to the proof of Theorem 4.1. $\square$

Corollary 5.1. Let $\theta \geq 0$ and let $p$ be a real number with $p>1$. Let $X$ be a normed vector space with norm $\|\cdot\|$. Let $f: X \rightarrow Y$ be a mapping satisfying $f(0)=0$ and

$$
N\left(f(m x+n y)-\frac{(m+n) f(x+y)}{2}-\frac{(m-n) f(x-y)}{2}, t\right) \geq \frac{t}{t+\theta\left(\|x\|^{p}+\|y\|^{p}\right)}(17)
$$

for all $x, y \in X$ and all $t>0$. Then

$$
A(x):=N-\lim _{n \rightarrow \infty} m^{n} f\left(\frac{x}{m^{n}}\right)
$$

exists for each $x \in X$ and defines an additive mapping $A: X \rightarrow Y$ such that

$$
N(f(x)-A(x), t) \geq \frac{\left(m^{p+1}-m^{2}\right) t}{\left(m^{p+1}-m^{2}\right) t+m \theta\|x\|^{p}} .
$$

Proof. The proof follows from Theorem 5.1 by taking

$$
\varphi(x, y):=\theta\left(\|x\|^{p}+\|y\|^{p}\right)
$$

for all $x, y \in X$. Then we can choose $L=m^{1-p}$ and we get the desired result. $\square$

Theorem 5.2. Let $\phi: X^{2} \rightarrow[0, \infty)$ be a function such that there exists an $L<1$ with

$$
\varphi(m x, m y) \leq m L \varphi(x, y)
$$

for all $x, y \in X$. Let $f: X \rightarrow Y$ be a mapping satisfying $f(0)=0$ and

$$
N\left(f(m x+n y)-\frac{(m+n) f(x+\gamma)}{2}-\frac{(m-n) f(x-y)}{2}, t\right) \geq \frac{t}{t+\phi(x, y)}
$$


for all $x, y \in X$ and all $t>0$. Then the limit

$$
R(x):=N-\lim _{n \rightarrow \infty} \frac{f\left(m^{n} x\right)}{m^{n}}
$$

exists for each $x \in X$ and defines an additive mapping $A: X \rightarrow Y$ such that

$$
N(f(x)-A(x), t) \geq \frac{(m-m L) t}{(m-m L) t+\varphi(x, 0)}
$$

for all $x, y \in X$ and all $t>0$.

Proof. The proof is similar to that of the proofs of Theorems 4.1 and 5.1. $\square$

Corollary 5.2. Let $\theta \geq 0$ and let $p$ be a real number with $0<p<1$. Let $X$ be $a$ normed vector space with norm $\|\cdot\|$. Let $f: X \rightarrow Y$ be a mapping satisfying $f(0)=0$ and

$$
N\left(f(m x+n y)-\frac{(m+n) f(x+y)}{2}-\frac{(m-n) f(x-y)}{2}, t\right) \geq \frac{t}{t+\theta\left(\|x\|^{p}+\|y\|^{p}\right)}
$$

for all $x, y \in X$ and all $t>0$. Then the limit

$$
A(x):=N-\lim _{n \rightarrow \infty} \frac{f\left(m^{n} x\right)}{m^{n}}
$$

exists for each $x \in X$ and defines a unique additive mapping $A: X \rightarrow Y$ such that

$$
N(f(x)-A(x), t) \geq \frac{\left(m-m^{p}\right) t}{\left(m-m^{p}\right) t+\theta\|x\|^{p}} .
$$

Proof. The proof follows from Theorem 5.2 by taking

$$
\varphi(x, y):=\theta\left(\|x\|^{p}+\|y\|^{p}\right)
$$

for all $x, y, z \in X$. Then we can choose $L=m^{p-1}$ and we get the desired result. $\square$

Example 5.1. Let $(Y, N)$ be a normed fuzzy Banach space. Let $f: Y \rightarrow Y$ be a mapping defined by

$$
f(z)=\left\{\begin{array}{l}
z, z \in\{m x+n y:\|m x+n y\|<1\} \cap\{x-y:\|x-y\|<1\} \\
0, \text { otherwise }
\end{array} .\right.
$$

Then one can easily show that $f: Y \rightarrow Y$ satisfies (5.2) for the case $p=1$ and that there does not exist an additive mapping satisfying (5.3).

\section{Conclusion}

We linked here five different disciplines, namely, the random normed spaces, nonArchimedean normed spaces, fuzzy normed spaces, functional equations, and fixed point theory. We established the Hyers-Ulam stability of the functional equation (1) in various normed spaces by using fixed point method.

Acknowledgements

The second author was supported by Basic Science Research Program through the National Research Foundation of Korea funded by the Ministry of Education, Science and Technology (NRF-2010-0013211). 


\section{Authors' contributions}

All authors conceived of the study, participated in its design and coordination, drafted the manuscript, participated in the sequence alignment, and read and approved the final manuscript.

\section{Competing interests}

The authors declare that they have no competing interests.

Received: 2 June 2011 Accepted: 25 October 2011 Published: 25 October 2011

\section{References}

1. Ulam, SM: Problems in Modern Mathematics. John Wiley and Sons, Science (1964)

2. Hyers, DH: On the stability of the linear functional equation. Proc Natl Acad Sci USA. 27, 222-224 (1941). doi:10.1073/ pnas.27.4.222

3. Rassias, ThM: On the stability of the linear mapping in Banach spaces. Proc Am Math Soc. 72, 297-300 (1978). doi:10.1090/S0002-9939-1978-0507327-1

4. Găvruta, P: A generalization of the Hyers-Ulam-Rassias stability of approximately additive mappings. J Math Anal Appl. 184, 431-436 (1994). doi:10.1006/jmaa.1994.1211

5. Skof, F: Local properties and approximation of operators. Rend Sem Mat Fis Milano. 53, 113-129 (1983). doi:10.1007/ BF02924890

6. Cholewa, PW: Remarks on the stability of functional equations. Aequationes Math. 27, 76-86 (1984). doi:10.1007/ BF02192660

7. Czerwik, S: Functional Equations and Inequalities in Several Variables. World Scientific, River Edge, NJ (2002)

8. Kenary, HA: The probabilistic stability of a Pexiderial functional equation in random normed spaces. Rend Del Circolo Math Di Palermo. (to appear)

9. Kenary, HA, Shafaat, Kh, Shafei, M, Takbiri, G: Hyers-Ulam-Rassias stability of the Appollonius quadratic mapping in RNspaces. J Nonlinear Sci Appl. 4, 110-119 (2011)

10. Park, C, Moradlou, F: Stability of homomorphisms and derivations on $C^{*}$-ternary rings. Taiwan J Math. 13, 1985-1999 (2009)

11. Saadati, R, Vaezpour, $M, C h o, Y$ : A note to paper "On the stability of cubic mappings and quartic mappings in random normed spaces". J Inequal Appl 2009 (2009). Article ID 214530

12. Saadati, R, Zohdi, MM, Vaezpour, SM: Nonlinear $\mathcal{L}$-random stability of an ACQ functional equation. J Inequal Appl 2011 (2011). Article ID 194394

13. Kenary, HA: On the Hyers-Ulam-Rassias stability of a functional equation in non-Archimedean and random normed spaces. Acta Universitatis Apulensis. (to appear)

14. Hensel, K: Ubereine news Begrundung der Theorie der algebraischen Zahlen. Jahresber Deutsch Math Verein. 6, 83-88 (1897)

15. Schewizer, B, Sklar, A: Probabilistic Metric Spaces. North-Holland Series in Probability and Applied Mathematics, NorthHolland, New York (1983)

16. Sherstnev, AN: On the notion of a random normed space. Dokl Akad Nauk SSSR 149, 280-283 (1963). (in Russian)

17. Bag, T, Samanta, SK: Finite dimensional fuzzy normed linear spaces. J Fuzzy Math. 11, 687-705 (2003)

18. Mirmostafaee, AK, Mirzavaziri, M, Moslehian, MS: Fuzzy stability of the Jensen functional equation. Fuzzy Sets Syst. 159 730-738 (2008). doi:10.1016/j.fss.2007.07.011

19. Bag, T, Samanta, SK: Fuzzy bounded linear operators. Fuzzy Sets Syst. 151, 513-547 (2005). doi:10.1016/j.fss.2004.05.004

20. Diaz, J, Margolis, B: A fixed point theorem of the alternative for contractions on a generalized complete metric space. Bull Am Math Soc. 74, 305-309 (1968). doi:10.1090/S0002-9904-1968-11933-0

21. Cădariu, L, Radu, V: Fixed points and the stability of Jensen's functional equation. J Inequal Pure Appl Math 4 (2003). Art ID 4

22. Mihet, $D, R a d u, V:$ On the stability of the additive Cauchy functional equation in random normed spaces. J Math Anal Appl. 343, 567-572 (2008)

doi:10.1186/1687-1812-2011-67

Cite this article as: Kenary et al:: A fixed point approach to the Hyers-Ulam stability of a functional equation in various normed spaces. Fixed Point Theory and Applications 2011 2011:67.

\section{Submit your manuscript to a SpringerOpen ${ }^{\circ}$ journal and benefit from:}

- Convenient online submission

Rigorous peer review

- Immediate publication on acceptance

- Open access: articles freely available online

- High visibility within the field

- Retaining the copyright to your article

Submit your next manuscript at $\gg$ springeropen.com 